\title{
High Sustained Virologic Response to Daclatasvir Plus Asunaprevir in Elderly and Cirrhotic Patients with Hepatitis C Virus Genotype 1b Without Baseline NS5A Polymorphisms
}

Fiona McPhee $\cdot$ Yoshiyuki Suzuki · Joji Toyota - Yoshiyasu Karino - Kasuaki Chayama

Yoshiiku Kawakami · Min Lung Yu · Sang Hoon Ahn · Hiroki Ishikawa · Rafia Bhore •

Nannan Zhou $\cdot$ Dennis Hernandez $\cdot$ Patricia Mendez $\cdot$ Hiromitsu Kumada

To view enhanced content go to www.advancesintherapy.com

Received: May 28, 2015 / Published online: July 9, 2015

(C) The Author(s) 2015. This article is published with open access at Springerlink.com

\section{ABSTRACT}

Introduction: Oral daclatasvir (DCV; pangenotypic NS5A inhibitor) plus asunaprevir (ASV; NS3 protease inhibitor) is approved in Japan and Korea for treatment of chronic hepatitis $\mathrm{C}$ virus (HCV) genotype 1 . Response to DCV + ASV is affected by DCV resistanceassociated polymorphisms (RAPs) in HCV NS5A. The prevalence and influence of these RAPs on 12-week sustained virologic response (SVR12) to

Electronic supplementary material The online version of this article (doi:10.1007/s12325-015-0221-5) contains supplementary material, which is available to authorized users.

F. McPhee $(\bowtie) \cdot$ N. Zhou $\cdot$ D. Hernandez Bristol-Myers Squibb Research and Development, Wallingford, CT, USA

e-mail: fiona.mcphee@BMS.com

Y. Suzuki · H. Kumada

Toranomon Hospital, Tokyo, Japan

J. Toyota $\cdot$ Y. Karino

Sapporo Kosei General Hospital, Sapporo, Japan

K. Chayama · Y. Kawakami

Hiroshima University, Hiroshima, Japan

M. L. Yu

Kaohsiung Medical University Hospital, Kaohsiung

Medical University, Kaohsiung, Taiwan
$\mathrm{DCV}+\mathrm{ASV}$ was evaluated in Asian and nonAsian patients.

Methods: Data were pooled from 5 national and international studies of patients with $\mathrm{HCV}$ genotype $1 b$ (GT-1b) receiving $\mathrm{DCV}+\mathrm{ASV}$ at their recommended doses. Baseline NS5A RAPs and their effect on SVR12 were assessed overall, in older ( $\geq 65$ years) patients, patients with cirrhosis, and in patients stratified by baseline HCV RNA or prior treatment experience with interferon-based therapy.

Results: Baseline NS5A sequences were available from 988 patients (374 Japanese; 125 Korean/Taiwanese; 489 from non-Asian countries), 979 of whom were assessed for

S. H. Ahn

Yonsei University College of Medicine, Seoul, South Korea

\section{H. Ishikawa}

Bristol-Myers KK, Tokyo, Japan

\section{R. Bhore - P. Mendez}

Bristol-Myers Squibb Research and Development, Princeton, NJ, USA 
SVR12. Pretreatment NS5A-L31F/I/M/V and/or NS5A-Y93H polymorphisms were present in $18 \%$ of Japanese and $12-13 \%$ of non-Japanese patients; these RAPs reduced SVR12 by $54.9 \%$ overall (93.9\% [787/838] SVR12 when absent, $39.0 \%$ [55/141] SVR12 when present), with comparable reductions observed in Asians and non-Asians and across all categories of treatment experience, age, and cirrhosis. RAPassociated SVR12 rates declined with increasing baseline HCV RNA (SVR12 with RAPs: $64.7 \%$ [11/17] at 5-6 $\log _{10} \mathrm{IU} / \mathrm{mL}, 29.8 \%$ [14/47] at 7-8 $\left.\log _{10}\right)$. Without baseline RAPs, very high SVR12 rates (92-100\%) were observed in older patients and patients with cirrhosis irrespective of national origin, with similarly high rates observed among treatment-naïve and interferon-experienced patients and those with high baseline HCV RNA.

Conclusions: Following DCV + ASV treatment, the SVR12 rates in GT-1b patients without baseline NS5A-L31F/I/M/V and/or NS5A-Y93H polymorphisms were very high (approximately 90-100\%), irrespective of age, cirrhosis, prior interferon treatment, or baseline HCV RNA.

Funding: Bristol-Myers Squibb.

Keywords: Asunaprevir; Daclatasvir; Drug resistance; Infectious diseases; Viral hepatitis

\section{INTRODUCTION}

Hepatitis C virus (HCV) is a highly polymorphic single-stranded RNA flavivirus that exists as 7 geographically diverse major genotypes with at least 67 subtypes [1]. Approximately, 80\% of acute infections with HCV fail to clear spontaneously and progress to a state of chronic infection with serious long-term sequelae. It is estimated that within 20 years of infection, $60-70 \%$ of people with untreated chronic $\mathrm{HCV}$ infection will develop hepatic steatosis or fibrosis, up to $20 \%$ will develop cirrhosis, and up to 5\% will progress to hepatocellular carcinoma [2]. Chronic HCV infection is estimated to be responsible for up to half a million liver-related deaths per annum [3].

Treatment of HCV has evolved and improved remarkably in recent years. The ongoing introduction of all-oral combination regimens of direct-acting antiviral (DAA) agents has resulted in significantly shorter treatment durations, better tolerability, and higher rates of post-treatment sustained virologic response (SVR), a surrogate of cure, than earlier treatments based on the use of parenteral interferons. One such all-oral regimen combines daclatasvir (DCV)—a pangenotypic inhibitor of the multifunctional HCV NS5A nonstructural protein that is active in vitro against HCV genotype 1 (GT-1) through GT-6 [4]-with asunaprevir (ASV), an NS3 protease inhibitor with in vitro activity against HCV GT1, GT-4, GT-5, and GT-6 [5]. In combination, treatment with these two agents has produced high rates of SVR among patients infected with the GT-1b subtype, including treatment-naïve patients, patients medically ineligible for-or intolerant of-peginterferon alfa and ribavirin (pegIFN/RBV), and patients who had previously not responded to or relapsed on pegIFN/RBV treatment [6-10]. The combination of DCV and ASV has been approved in Japan and Korea for the treatment of HCV genotype GT-1 and is currently under regulatory evaluation in other countries.

The HCV pandemic in Asia differs from that in the West in several ways that may impact response to DCV + ASV or other new combinations of DAAs. Across much of Asia, the GT-1b subtype of GT-1 is the predominant HCV strain, whereas in North America and Northern Europe GT-1a is by far the most 
common [2]. The spread of HCV in Asia also predates that in North America and Western Europe, resulting in a significantly higher proportion of elderly patients in countries such as Japan and Taiwan than in most nonAsian countries, and a correspondingly higher incidence of cirrhosis and hepatocellular carcinoma [11]. Both older age and cirrhosis are historically associated with a higher burden of adverse events and poorer responses to interferon-based therapy $[12,13]$, and their influence on response to newer oral regimens remains an area of great interest and research.

Treatment-emergent NS5A variants associated with drug resistance and virologic failure have been described for DCV and other inhibitors of HCV NS5A, and these may exist pretreatment as naturally occurring resistanceassociated polymorphisms (RAPs) whose prevalence may vary by region and subtype. Treatment-emergent variants at NS5A amino acid positions 31 and 93 have been observed in both GT-1a and GT-1b virologic failures receiving DCV-containing regimens [14-17]. NS5A variants at these amino acid positions can also exist pre-therapy. In HCV GT-1binfected Japanese patients, pre-existence of NS5A RAPs at L31 or $\mathrm{Y} 93 \mathrm{H}$, as determined by a population-based direct sequencing approach, has impacted SVR to DCV + ASV [17]. Two additional treatment-emergent NS5A variants at amino acid positions 28 and 30 have also been observed in patients with GT-1a virologic failure receiving DCV-containing regimens, which may also be present as RAPs at pretherapy baseline $[14,15]$.

Effective use of DCV + ASV as a treatment option in Asia requires an understanding of how RAPs and other influences of importance to Asian populations, such as older age and cirrhosis, affect treatment outcomes. The prevalence of pre-therapy NS5A polymorphisms, their influence on SVR to DCV + ASV treatment, and their interactions with other potential baseline predictors of response in both Asian and Non-Asian patients was evaluated in pooled data from the $\mathrm{DCV}+\mathrm{ASV}$ clinical development program.

\section{METHODS}

\section{Studies and Patients}

Data were pooled from all 5 phase 2 and phase 3 clinical studies of DCV + ASV in patients with chronic HCV GT-1 infection undertaken as part of the DCV + ASV development program. The studies included have all been previously reported and comprised: AI447-017 (Clinical Trials.gov number, NCT01012895; phase 2) [7] and AI447-026 (ClinicalTrials.gov number, NCT01497834; phase 3) [8] evaluating $\mathrm{DCV}+\mathrm{ASV}$ in Japanese patients with prior non-response to pegIFN/RBV, or who were intolerant of or medically ineligible for pegIFN/RBV; AI447-028 (HALLMARK-DUAL; ClinicalTrials.gov number, NCT01581203) [9], a multinational (excluding Japan) phase 3 evaluation of DCV + ASV in patients who were treatment-naïve, were prior non-responders to pegIFN/RBV, or were intolerant/ineligible for pegIFN/RBV; and AI447-031 (ClinicalTrials.gov number, NCT01718145) [10], a Japanese phase 3 study of DCV + ASV in patients who were treatment-naïve or who had previously undergone virologic relapse following pegIFN/ RBV treatment. In addition, a subset of patients were included who received $\mathrm{DCV}+\mathrm{ASV}$ in study AI447-011 (ClinicalTrials.gov number, NCT01012895) [6], a phase 2 evaluation of $\mathrm{DCV}+\mathrm{ASV}, \quad \mathrm{DCV}+\mathrm{ASV}+\operatorname{pegIFN} / \mathrm{RBV}, \quad$ and $\mathrm{DCV}+\mathrm{ASV}+\mathrm{RBV}$ in prior non-responders to pegIFN/RBV from France, Puerto Rico, and the United States. 
Patients included in these analyses were infected with HCV subtype GT-1b only and had been assigned to receive 24 weeks of $\mathrm{DCV}+\mathrm{ASV}$ at their recommended doses of $60 \mathrm{mg}$ DCV once daily with either $200 \mathrm{mg}$ (phase 2 dry tablet formulation) or $100 \mathrm{mg}$ (phase 3 soft-gel formulation) ASV twice daily. Patients had at least 12 weeks of post-treatment follow-up. Patients with compensated cirrhosis who fulfilled the other criteria were included from the 2 studies (AI447-026 and AI447-028) that enrolled cirrhotic subjects. Cirrhosis was defined by pretreatment biopsy, or by either a baseline FibroScan result $\geq 14.6 \mathrm{kPa}$ in AI447028 or according to the published laboratory algorithm of Ikeda et al. [18] in AI447-026.

\section{Evaluations and Analyses}

Baseline polymorphisms in HCV NS5A present in approximately $20 \%$ or more of the patients' virus population were assessed in each study by population-based sequencing as previously described [17] using the Con1 GT-1b consensus sequence as a reference (Accession number: AJ238799). Briefly, PCR-amplified NS5A sequences were cloned using a TOPO-TA Cloning Kit (Invitrogen, Carlsbad, CA, USA) and sequenced using the BigDye Terminator v3.1 Cycle Sequencing Kit (Applied Biosystems, South San Francisco, CA, USA). Individual reactions were purified using the BigDye XTerminator Purification Kit (Applied Biosystems) before being loaded on an Applied Biosystems DNA Analyzer 3730XL. Nonconsensus changes at amino acid positions 28 (L28M), 30 (R30Q), 31 (L31F/I/M/V), and 93 (Y93H) known to be associated with resistance-associated GT-1 virologic failures on DCV were included in analyses of prevalence and virologic outcome.

The primary virologic outcome for these analyses was SVR at post-treatment week 12
(SVR12), which was assessed centrally in each study and defined as HCV RNA below the lower limit of assay quantitation (LLOQ) for the assay used in that study, with or without detectable target. Japanese studies AI447-017, AI447-026, and AI447-031 measured HCV RNA using the Roche COBAS TaqMan HCV Auto assay (Roche Diagnostics KK, Tokyo, Japan) with an LLOQ of 15 IU/mL. The non-Japanese studies AI447-011 and AI447-028 used the COBAS TaqMan HCV test version 2.0 (Roche Molecular Systems, Pleasanton, CA, USA) with an LLOQ of $25 \mathrm{IU} /$ $\mathrm{mL}$. Missing virologic data at post-treatment week 12 (i.e., patient missing SVR12 outcome) was imputed from the next available posttreatment HCV RNA measurement via a nextobservation-carried-backward approach. Late relapsers $(<\mathrm{LLOQ}$ at post-treatment week 12 but subsequently $\geq$ LLOQ at a later posttreatment measurement) were classed as having had SVR12 failure.

Two populations were derived for the analyses. The full population comprised all patients in the 5 studies who had available baseline NS5A sequence data and is the set in which the prevalence of baseline NS5A polymorphisms was assessed. The SVR12 population comprised a subset of the full population that excluded patients with nonvirologic treatment failures and is the set in which the effects of polymorphisms and other baseline factors on SVR12 was assessed. Patients with non-virologic failure had achieved an SVR at post-treatment week 4 but had no subsequent follow-up data, or had received fewer than 84 days (12 weeks) of DCV + ASV treatment and discontinued for reasons other than lack of efficacy.

For both prevalence and SVR12 analyses, patients were categorized by country of residence and grouped as "Japanese," "Korean/ Taiwanese," and from "Non-Asian countries." 
Three studies (AI447-017, AI447-026, and AI447-031) were specific to Japan, making Japanese patients the single largest Asian group. Smaller numbers of Asian patients from Korea and Taiwan were enrolled into AI447028, which did not enroll patients in Japan, and were pooled for analysis as a separate Asian group. The "Non-Asian countries" group consisted of all others from AI447-028 and AI447-011 and comprised patients from Argentina, Australia, Austria, Canada, France, Germany, Ireland, Israel, Italy, the Netherlands, Poland, Russia, Spain, the United Kingdom, and the United States. Patients were further stratified for SVR12 analysis by prior treatment status, cirrhosis status, age, baseline HCV RNA, and the presence of NS5A polymorphisms at $\mathrm{L} 31$ and $\mathrm{Y} 93 \mathrm{H}$.

All analyses were descriptive, without formal significance testing. The analyses described are based on data from earlier studies, described elsewhere, that were conducted in accordance with all relevant national and local ethical and regulatory requirements and in accordance with the Helsinki Declaration of 1964 and its subsequent revisions. No new studies of human or animal subjects were performed for these analyses.

\section{RESULTS}

\section{Patient Demographics}

Table 1 summarizes the 5 studies included in these pooled analyses and the number of patients from each who were included in the assessments of NS5A polymorphisms and SVR12 outcomes. In total, 988 patients were included in the full population, of whom 979 formed the SVR12 population. The 9 patients in the full population who were excluded from the SVR12 population comprised 2 patients (studies AI447-017 and AI447-028) who did not meet study criteria for resistance testing at failure due to having HCV RNA $<$ LLOQ at their last visits; 2 patients (studies AI447-026 and AI447-028) who had requested that treatment be discontinued; 2 patients in AI447-028 who withdrew consent; 1 patient in AI447-028 who was lost to follow-up; and 2 patients in AI447026 who discontinued therapy due to an adverse event.

Table 1 Studies included in the pooled analyses

\begin{tabular}{|c|c|c|c|c|c|c|}
\hline Study & $\begin{array}{l}\text { ClinicalTrials. } \\
\text { gov ID }\end{array}$ & Phase & Patients & $\begin{array}{l}\text { Analyzed } \\
\text { for } \\
\text { BL NS5A }\end{array}$ & $\begin{array}{l}\text { Analyzed } \\
\text { for } \\
\text { SVR12 }\end{array}$ & References \\
\hline AI447-011 & NCT01012895 & 2 & Non-responders ${ }^{\mathrm{a}}$ & 17 & 17 & Lok et al. [6] \\
\hline AI $447-017^{\mathrm{b}}$ & NCT01051414 & 2 & $\begin{array}{l}\text { Non-responders }{ }^{\mathrm{a}} \text { or IFN/RBV } \\
\text { intolerant or ineligible }\end{array}$ & 33 & 32 & Suzuki et al. [7] \\
\hline AI $447-026^{b}$ & NCT01497834 & 3 & $\begin{array}{l}\text { Non-responders or IFN/RBV } \\
\text { intolerant or ineligible }\end{array}$ & 214 & 211 & Kumada et al. [8] \\
\hline AI447-028 & NCT01581203 & 3 & $\begin{array}{l}\text { Naïve, non-responders, or IFN/ } \\
\text { RBV intolerant or ineligible }\end{array}$ & 597 & 592 & Manns et al. [9] \\
\hline AI $447-031^{b}$ & NCT01718145 & 3 & Naïve and IFN relapsers & 127 & 127 & Chayama et al. [10] \\
\hline \multicolumn{7}{|c|}{$\begin{array}{l}B L \text { baseline, } I F N \text { interferon, } R B V \text { ribavirin } \\
\text { a All enrolled in this category were null responders } \\
\text { b Japan-only studies }\end{array}$} \\
\hline
\end{tabular}


Table 2 Baseline characteristics

\begin{tabular}{|c|c|c|c|c|}
\hline Characteristic & $\begin{array}{l}\text { Treatment naïve } \\
(N=296)\end{array}$ & $\begin{array}{l}\text { Non-responders } \\
(N=304)\end{array}$ & $\begin{array}{l}\text { IFN/RBV intolerant or } \\
\text { ineligible }(N=371)\end{array}$ & Relapsers $(N=17)$ \\
\hline \multicolumn{5}{|l|}{ Age } \\
\hline Mean (range), years & $53.4(20-79)$ & $56.7(23-77)$ & $59.4(24-77)$ & $61.4(45-75)$ \\
\hline$<65$ years, $n(\%)$ & $254(85.8)$ & $235(77.3)$ & $248(66.8)$ & $10(58.8)$ \\
\hline$\geq 65$ years, $n(\%)$ & $42(14.2)$ & $69(22.7)$ & $123(33.2)$ & $7(41.2)$ \\
\hline Male, $n(\%)$ & $138(46.6)$ & $159(52.3)$ & $140(37.7)$ & $7(41.2)$ \\
\hline \multicolumn{5}{|l|}{ Countries, $n(\%)$} \\
\hline Japan & $110(37.2)$ & $97(31.9)$ & $150(40.4)$ & $17(100)$ \\
\hline Korea & $21(7.1)$ & $20(6.6)$ & $22(5.9)$ & 0 \\
\hline Taiwan & $19(6.4)$ & $21(6.9)$ & $22(5.9)$ & 0 \\
\hline Non-Asian & $146(49.3)$ & $166(54.6)$ & $177(47.7)$ & 0 \\
\hline \multicolumn{5}{|l|}{ HCV RNA } \\
\hline Mean (range), $\log _{10} \mathrm{IU} / \mathrm{mL}$ & $6.5(4.0-8.0)$ & $6.6(4.0-8.0)$ & $6.4(4.0-8.0)$ & $7.1(6.0-8.0)$ \\
\hline Cirrhotic patients, $n(\%)$ & $31(10.5)$ & $71(23.4)$ & $115(31.0)$ & 0 \\
\hline \multicolumn{5}{|c|}{ Baseline NS5A polymorphisms, $n(\%)$} \\
\hline $\mathrm{L} 31 \mathrm{~F} / \mathrm{I} / \mathrm{M} / \mathrm{V}$ & $13(4.4)$ & $18(5.9)$ & $11(3.0)$ & $1(5.9)$ \\
\hline $\mathrm{Y93H}$ & $26(8.8)$ & $25(8.2)$ & $52(14.0)$ & $1(5.9)$ \\
\hline
\end{tabular}

$H C V$ hepatitis $\mathrm{C}$ virus, $I F N$ interferon, $R B V$ ribavirin

The full population (Table 2) comprised 296 treatment-naïve patients, 304 non-responders to pegIFN/RBV, 371 patients unable to be treated with pegIFN/RBV for reasons of intolerance or medical ineligibility, and 17 prior relapsers on pegIFN/RBV. There were 374 Japanese patients (37.9\% of total), 63 Koreans (6.4\%), 62 Taiwanese (6.3\%), and 489 patients (49.5\%) from non-Asian countries.

\section{Prevalence of NS5A Polymorphisms}

The prevalence of NS5A baseline polymorphisms in the full population is shown in Fig. 1. With the exception of amino acid 31, polymorphisms at the positions of interest generally appeared to be more common among Asian than non-Asian patients. In particular, the incidence of NS5AY93H with or without L31F/I/M/V polymorphisms was higher among Japanese patients than among Koreans, Taiwanese, or patients from non-Asian countries. When stratified by age, the incidence of $\mathrm{Y93H}$ remained higher among both younger and older Japanese patients than among the other Asians and non-Asians: $13.6 \%(34 / 250)$ in Japanese patients $<65$ years old and $17.7 \%(22 /$ $124)$ in Japanese patients $\geq 65$ years, versus $9.6 \%$ (9/94) and 12.9\% (4/31), respectively, among Koreans and Taiwanese, and 7.4\% (30/403) and $5.8 \%$ (5/86), respectively, among non-Asians. The prevalence of $\mathrm{Y93H}$ was higher in older than in younger Asian patients (overall 16.8\% [26/155] in Japanese, Koreans and Taiwanese $\geq 65$ years old, versus $12.5 \%$ [43/344] in those 


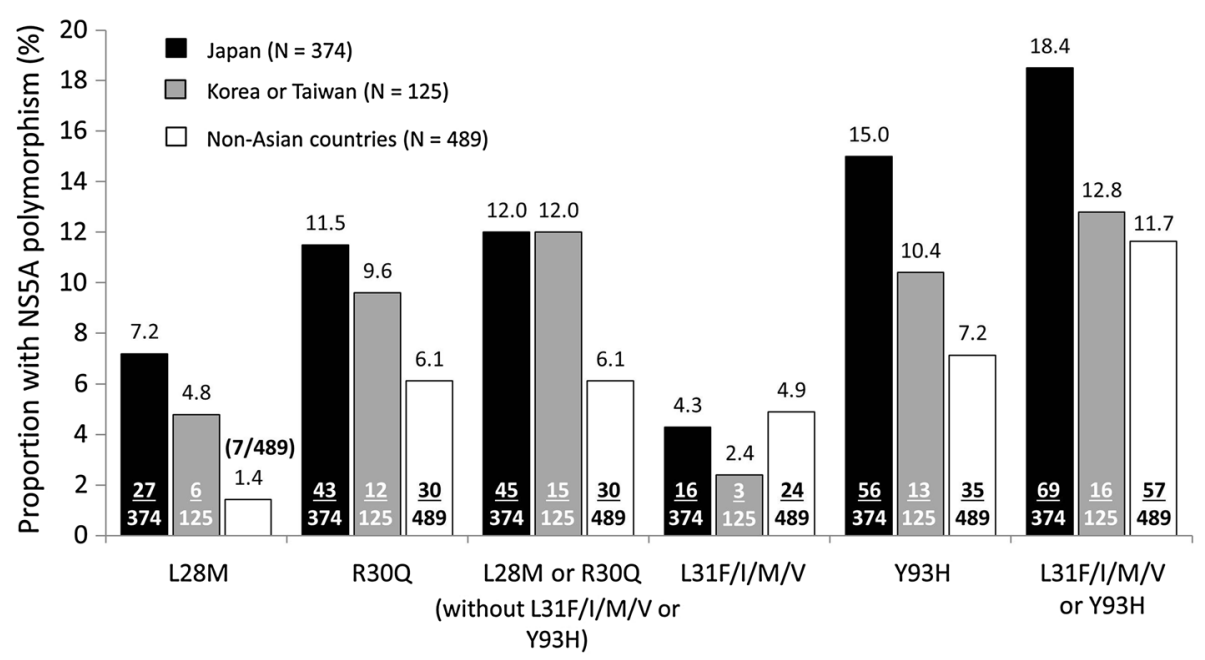

Fig. 1 Prevalence of baseline NS5A polymorphisms in Hepatitis C virus genotype $1 \mathrm{~b}$

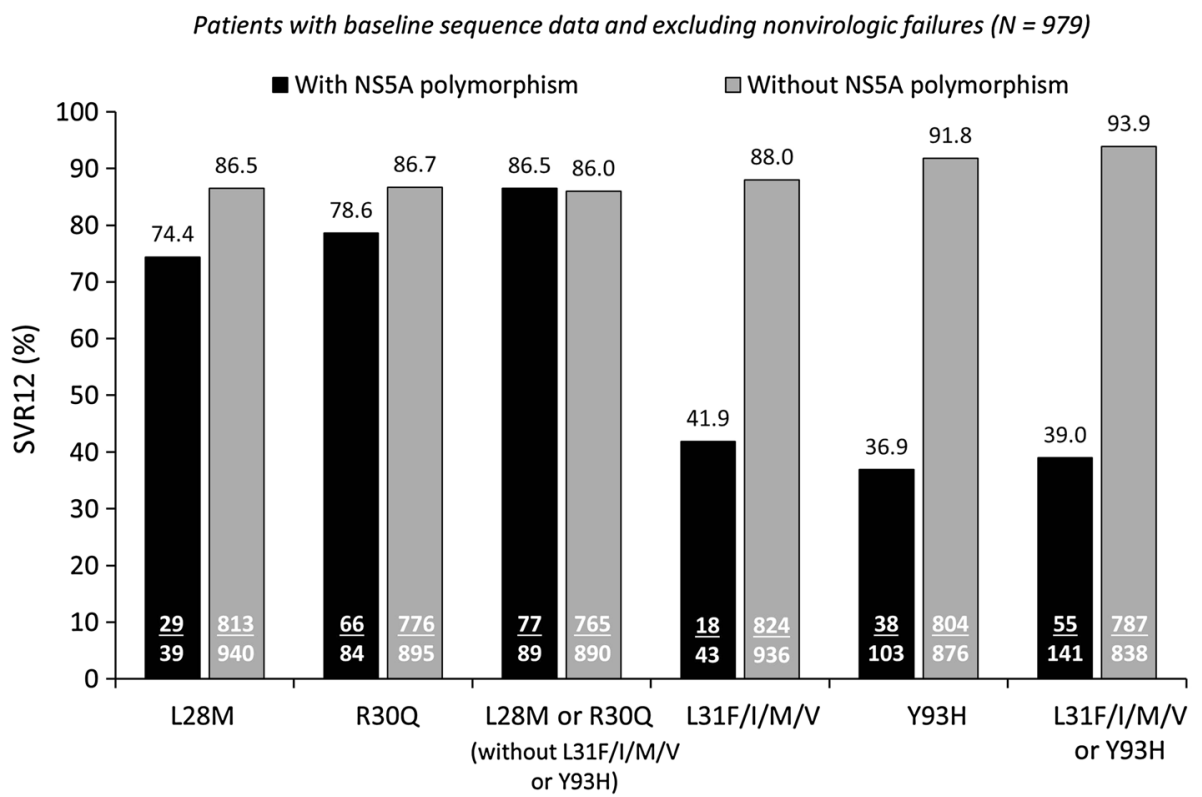

Fig. 2 SVR12 with versus without baseline NS5A polymorphisms. SVR12 12-week sustained virologic response

under 65 years), whereas the prevalence in older non-Asians (5.8\%) was similar to or slightly lower than that in younger patients (7.4\%).

\section{Effect of NS5A Polymorphisms and Other Baseline Factors on SVR12}

The association between baseline NS5A polymorphisms and SVR12 is shown in Fig. 2 for the full SVR12 population of 979 patients. Among all GT-1b-infected patients, the presence of the DCV NS5A RAPs L31F/I/M/V and/or $\mathrm{Y} 93 \mathrm{H}$ at baseline was associated with reduced SVR12 following treatment with DCV + ASV (SVR12 range: 36.9-41.9\%), while SVR12 rates in the absence of these RAPs were high (SVR12 range: 88.0-93.9\%). By contrast, neither baseline L28 M nor R30Q had any 
With L31F/I/M/V and/or Y93H

(i) Japan ( $\mathbf{N}=370)$

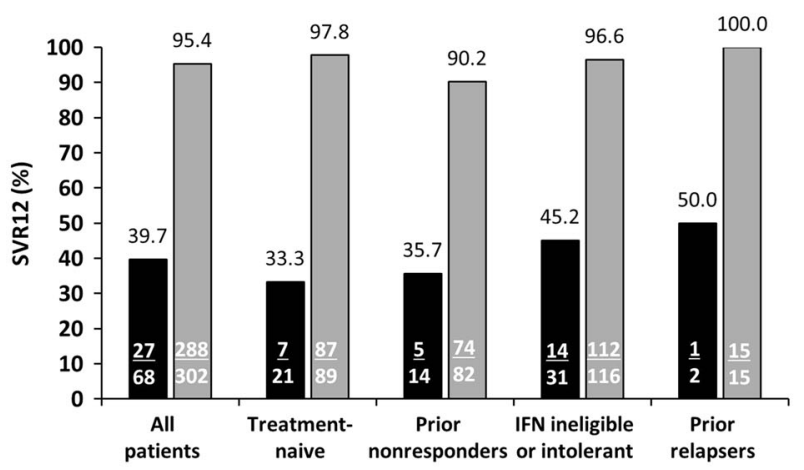

(iii) Non-Asian countries ( $\mathrm{N}=485$ )

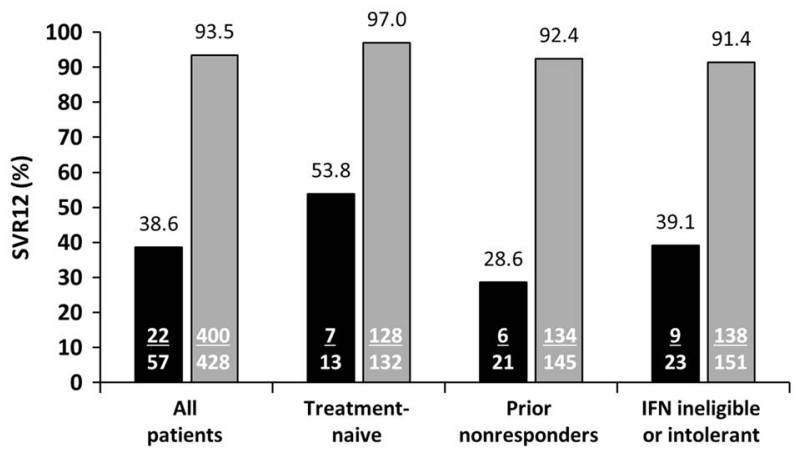

Without L31F/I/M/V or Y93H

(ii) Korea and Taiwan ( $\mathrm{N}=124)$

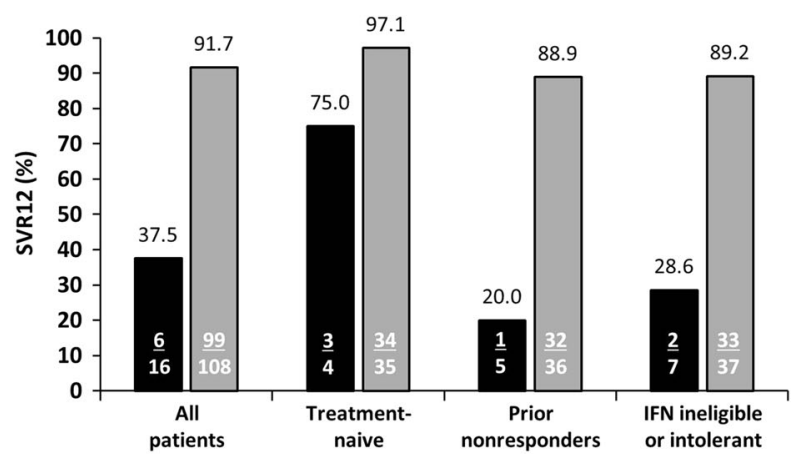

Fig. 3 SVR12 by prior treatment status and presence of baseline NS5A polymorphisms. IFN Interferon, SVR12 12-week sustained virologic response

relevant effect on SVR12 in the absence of NS5A polymorphisms at amino acids 31 or 93 (SVR12 approximately $86 \%$ ).

In contrast to the presence of NS5A-L31F/I/ $\mathrm{M} / \mathrm{V}$ or NS5A-Y93H, prior treatment status, older age ( $\geq 65$ years), and the presence of compensated cirrhosis did not influence SVR12 in either Asian or non-Asian patients. Figure 3 shows SVR12 by prior treatment status and presence of NS5A RAPs, and Fig. 4 shows SVR12 by age, cirrhosis status, and presence of NS5A RAPs for each national grouping. In the absence of baseline NS5A RAPs, SVR12 rates ranged from $89 \%$ to $100 \%$ across prior treatment categories, and were above 90\% irrespective of age or cirrhosis. There was no evidence of a difference in SVR12 outcomes between Japanese, non-Japanese Asians and patients from non-Asian countries. The declines in SVR12 among those with baseline NS5A RAPs versus those without were broadly similar across each baseline category, with no evidence of an interaction.

Figure 5 shows SVR12 by categories of baseline HCV RNA from $<5 \log _{10}$ to $7-8 \log _{10}$ $\mathrm{IU} / \mathrm{mL}$ and the presence of NS5A RAPs for all 979 patients in the SVR12 population. SVR12 rates declined with increasing viral load, with a slight reduction among patients without baseline NS5A RAPs, and a more substantial reduction among those with baseline L31 or Y93H polymorphisms. 
With L31F/I/M/V and/or Y93H

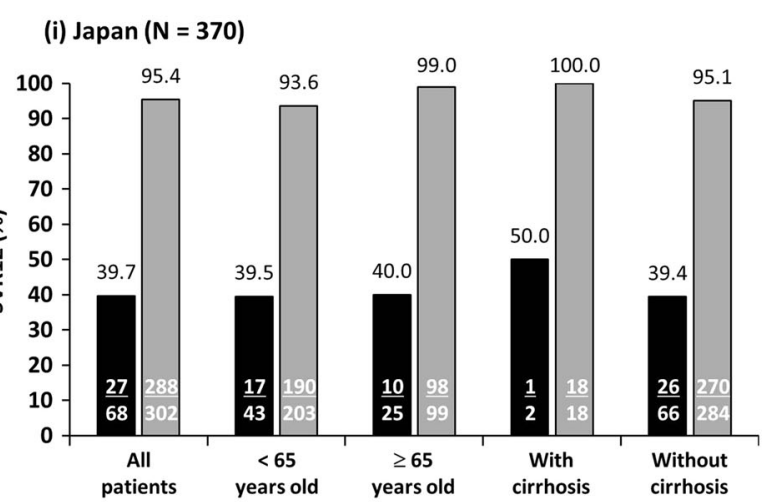

(iii) Non-Asian countries ( $\mathrm{N}=485)$

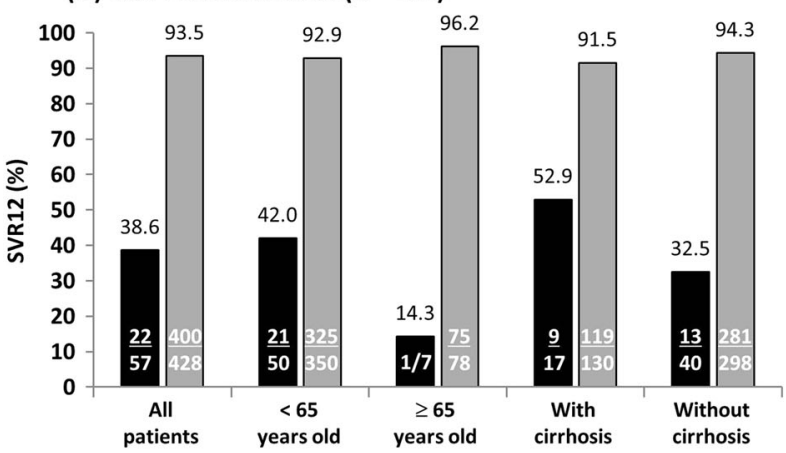

Without L31F/I/M/V or Y93H

(ii) Korea and Taiwan ( $\mathrm{N}=124)$

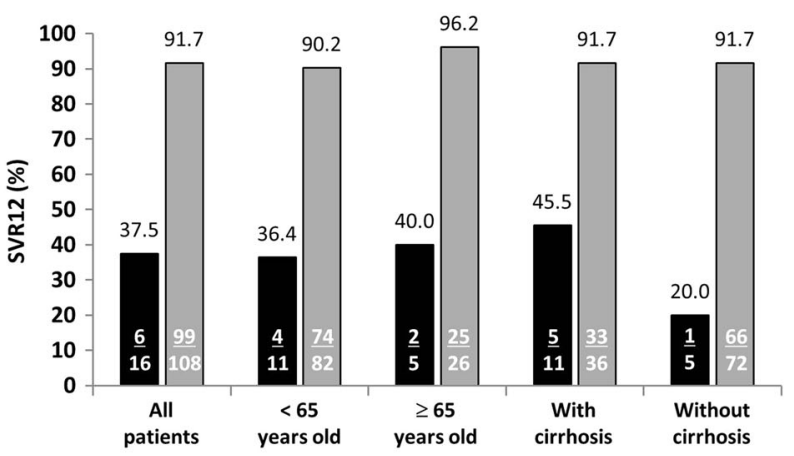

Fig. 4 SVR12 by age, cirrhosis status, and presence of baseline NS5A polymorphisms. SVR12 12-week sustained virologic response

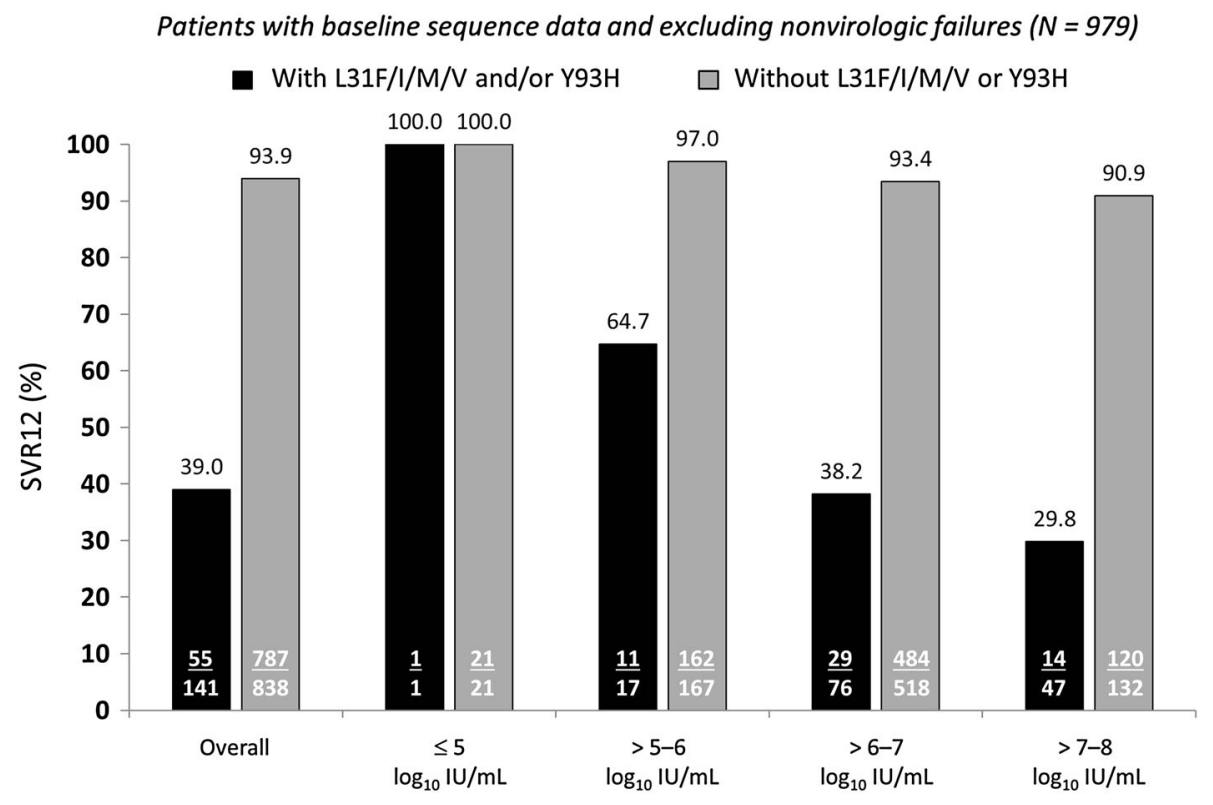

Baseline HCV RNA

Fig. 5 SVR12 by baseline HCV RNA and presence of baseline NS5A polymorphisms. HCV Hepatitis C virus, SVR12 12-week sustained virologic response 


\section{DISCUSSION}

Pretreatment polymorphisms at NS5A amino acid positions 31 or 93 in HCV GT-1b have been previously shown by Karino et al. [17] to reduce SVR to DCV + ASV by approximately $50 \%$ in a small sample of Japanese patients in study AI447-017. These findings were confirmed in this large, pooled dataset of patients including study AI447-017 and 4 additional studies, with the pre-existence of $\mathrm{L} 31 \mathrm{~F} / \mathrm{I} / \mathrm{M} / \mathrm{V}$ or $\mathrm{Y} 93 \mathrm{H}$ at baseline resulting in an overall 55\% reduction in SVR12, from 94\% to 39\%. These data also established a higher prevalence of L31 or $\mathrm{Y93H}$ polymorphisms in the Japanese GT-1b population $(18 \%)$ than in the non-Japanese patients (12-13\%). This difference was driven by the prevalence of $\mathrm{Y} 93 \mathrm{H}$, which was twice as common among Japanese patients (15\%) than those from non-Asian countries (7\%), and 50\% more common than among Asian patients from Korea and Taiwan (10\%). The difference in $\mathrm{Y} 93 \mathrm{H}$ prevalence between Japanese and other Asian patients was primarily due to a higher prevalence in Japanese patients under 65 years old than in younger Koreans and Taiwanese (13.6\% versus $9.6 \%)$, but with an additional contribution from the higher proportion of older ( $\geq 65$ years) patients in the Japanese group (33\%) compared to the non-Japanese Asians (25\%) amongst whom the prevalence of Y93H was also higher (17.7\% in older Japanese versus $12.9 \%$ in older Koreans and Taiwanese).

Interestingly, baseline polymorphisms at NS5A amino acid positions 28 or 30, previously associated with GT-1a virologic failures to DCV, were also twice as prevalent in both GT-1b Japanese and non-Japanese Asians (12\%) compared with non-Asians (6\%). However, these polymorphisms had no effect on SVR12 outcomes and therefore cannot be considered RAPs for GT-1b.
Among the key baseline characteristics of age, cirrhosis status, prior pegIFN/RBV treatment experience, and the presence of NS5A RAPs at L31 and Y93H, only the NS5A RAPs reduced SVR12. In the absence of L31F/I/ $\mathrm{M} / \mathrm{V}$ or $\mathrm{Y93H}$, very high SVR12 rates, typically between $90 \%$ and $100 \%$, were observed for all categories and with no obvious differences between Asian and non-Asian patient groups. Indeed, among those without baseline NS5A RAPs, $99 \%$ of elderly Japanese patients and $100 \%$ of Japanese patients with cirrhosis achieved SVR12 following DCV + ASV treatment, along with similarly high proportions (92-96\%) of elderly and cirrhotic patients without RAPs from Korea and Taiwan or from the non-Asian countries.

The only baseline characteristic examined that had an effect on SVR12 independent of baseline NS5A RAPs was HCV RNA, where a small but continuous decline in SVR12 from $100 \%$ to $91 \%$ was evident across baseline strata ranging from $<5 \log _{10} \mathrm{IU} / \mathrm{mL}$ to $7-8 \log _{10} \mathrm{IU} /$ $\mathrm{mL}$. This was also the only baseline characteristic that appeared to show an interaction with the presence of NS5A RAPs. In the absence of NS5A RAPs, a reduction in SVR12 rate of only $9 \%$ across all RNA strata was observed; however, in the presence of NS5A RAPs, reductions in SVR12 of approximately $50 \%$ were observed, with SVR12 rates ranging from $65 \%$ with a baseline viremia of 5-6 logs to $30 \%$ with 7-8 logs. The data suggest that the effects of NS5A-L31 and NS5A-Y93H polymorphisms on response to DCV + ASV are partially mitigated by low baseline viremia.

It is relevant to note that reduced baseline susceptibility to DCV may also be mitigated by the composition of the DCV-containing regimen. Reduced $\mathrm{HCV}$ susceptibility at baseline to any given antiviral may in principle be compensated using that antiviral 
in combination with several non-cross-resistant agents, and/or in combination with potent agents with a high barrier to resistance. The significant predictive effect of NS5A L31 and Y93H RAPs on reduced response to DCV + ASV shown here and in the data from Karino et al. [17] has not been apparent where DCV + ASV is used in combination with the non-nucleoside NS5B-inhibitor beclabuvir (BCV), or in studies of DCV with the nucleotide NS5B-inhibitor sofosbuvir. In the UNITY-1 (ClinicalTrials.gov number, NCT01979939) [19] and UNITY-2 (ClinicalTrials.gov number, NCT01973049) [20] studies of 12 weeks of treatment with $\mathrm{DCV}+\mathrm{ASV}+\mathrm{BCV}$ for GT-1 infection in patients without (UNITY-1) or with (UNITY-2) compensated cirrhosis, SVR12 was achieved by $100 \%$ of GT-1b-infected patients in both studies who had baseline NS5A RAPs at positions 28, 30, 31, or 93 (17 in UNITY-1, 13 in UNITY 2). In comparison, SVR12 among GT-1a-infected patients with baseline RAPs at these positions was 74\% [25/34] in UNITY-1 and 87\% [13/15] in UNITY-2. Similarly, in study AI444-040 evaluating DCV plus sofosbuvir with or without ribavirin, only 1 patient among 28 with GT-1, GT-2, or GT-3 infection and baseline NS5A-L31M or NS5A-Y93H/C/N failed to achieve SVR12, and this patient achieved SVR4 before being lost to follow-up [21]. These clinical data are consistent with in vitro observations of the antiviral activity of DCV plus sofosbuvir or $\mathrm{DCV}+\mathrm{ASV}+\mathrm{BCV}$ in $\mathrm{DCV}$ and ASV-resistant GT-1b subgenomic replicon cell lines bearing NS5A-L31M, NS5A-Y93H and NS3-D168V variants [22]. Thus, the clinical relevance of any given RAP or combination of RAPs on HCV response to a given DAA regimen cannot necessarily be extrapolated to other combinations involving the affected drugs.

While there are many baseline factors that could influence sustained $\mathrm{HCV}$ responses to a given regimen, overall these data suggest that pre-therapy screening for NS5A polymorphisms at $\mathrm{L} 31$ and $\mathrm{Y93H}$ may be beneficial in patients with HCV GT-1b infection eligible for treatment with DCV + ASV. Although this was a descriptive study without formal statistical testing, very high response rates to DCV + ASV of $90-100 \%$ were observed for patients where these NS5A polymorphisms were not detected by population-based sequencing, while patients with these NS5A polymorphisms were less likely to respond to this combination and may therefore obtain greater benefit from a different DCV-based combination. As discussed above, there are clinical data to suggest that GT1b-infected patients with baseline NS5A polymorphisms at $\mathrm{L} 31$ or $\mathrm{Y93H}$ will respond well to DCV plus sofosbuvir [21], or DCV + ASV + BCV $[19,20]$. There appears to be an ameliorating effect of low baseline HCV RNA on the influence of baseline NS5A polymorphisms at $\mathrm{L} 31$ and/or $\mathrm{Y} 93 \mathrm{H}$ on sustained response to $\mathrm{DCV}+\mathrm{ASV}$, but it is a limitation of the study that the number of patients with low HCV RNA and these polymorphisms is too low to establish a robust threshold of effect for clinical decision making.

\section{CONCLUSIONS}

Pooled data from clinical studies of DCV + ASV for chronic HCV GT-1b infection show that the most significant baseline correlate of SVR so far observed is the presence of pre-therapy DCV RAPs at NS5A amino acids L31 and/or Y93H. In the absence of these NS5A RAPs, very high rates of SVR12, between $90 \%$ and $100 \%$, were achieved, irrespective of older age, cirrhosis, prior experience of pegIFN/RBV, or baseline HCV RNA level. These data are of relevance to physicians in Asian countries where DCV + ASV is approved for HCV treatment. 


\section{ACKNOWLEDGMENTS}

All studies described and the analyses presented were sponsored by Bristol-Myers Squibb. The article processing charges and open access fee for this publication were funded by BristolMyers Squibb. All named authors meet the International Committee of Medical Journal Editors (ICMJE) criteria for authorship for this manuscript, take responsibility for the integrity of the work as a whole, and have given final approval to the version to be published. Editorial assistance with the preparation of this manuscript was provided by Nick Fitch, PhD, CMPP, of Articulate Science (London, UK), with funding from Bristol-Myers Squibb.

Conflict of interest. Fiona McPhee, Hiroki Ishikawa, Rafia Bhore, Nannan Zhou, Dennis Hernandez, and Patricia Mendez are all employees of Bristol-Myers Squibb and may be stockholders thereof. Kasuaki Chayama is a consultant/advisor for Abbvie and has received grant support from Bristol-Myers Squibb, AstraZeneca, GlaxoSmithKline, Janssen, and Merck Sharp \& Dohme, and speaker honoraria from Bristol-Myers Squibb, GlaxoSmithKline, and Merck Sharp \& Dohme. Min Lung Yu is a consultant for Merck Sharp \& Dohme, Abbott, and Abbvie, has received grant support from Abbott and Roche, and has been on speakers bureaus for Bristol-Myers Squibb, Roche, Merck Sharp \& Dohme, GlaxoSmithKline, Novartis, and Gilead Sciences. Yoshiyuki Suzuki, Joji Toyota, Yoshiyasu Karino, Yoshiiku Kawakami, Sang Hoon Ahn, and Hiromitsu Kumada declare that they have no conflict of interest.

Compliance with ethics guidelines. The analyses herein are based on previously conducted studies described elsewhere and do not involve any new studies of human or animal subjects performed by the authors. All five studies from which these data were drawn were conducted in accordance with the ethical standards of the responsible committee on human experimentation (institutional and national) and with the Helsinki Declaration of 1964, as revised in 2013. Informed consent was obtained from all patients prior to initiation of study procedures.

Open Access. This article is distributed under the terms of the Creative Commons Attribution Noncommercial License which permits any noncommercial use, distribution, and reproduction in any medium, provided the original author(s) and the source are credited.

\section{REFERENCES}

1. Smith DB, Bukh J, Kuiken C, et al. Expanded classification of hepatitis $\mathrm{C}$ virus into 7 genotypes and 67 subtypes: updated criteria and genotype assignment Web resource. Hepatology. 2014;59:318-27.

2. Ansaldi F, Orsi A, Sticchi L, Bruzzone B, Icardi G. Hepatitis $C$ virus in the new era: perspectives in epidemiology, prevention, diagnostics and predictors of response to therapy. World J Gastroenterol. 2014;20:9633-52.

3. World Health Organization. Hepatitis C Key Facts. WHO factsheet No 164. 2014. http://www.who.int/ mediacentre/factsheets/fs164/en/. Accessed Apr 2015.

4. Gao M, Nettles RE, Belema M, et al. Chemical genetics strategy identifies an HCV NS5A inhibitor with a potent clinical effect. Nature. 2010;465:96-100.

5. McPhee F, Sheaffer AK, Friborg J, et al. Preclinical profile and characterization of the hepatitis $\mathrm{C}$ virus NS3 protease inhibitor asunaprevir (BMS-650032). Antimicrob Agents Chemother. 2012;56:5387-96.

6. Lok AS, Gardiner DF, Hezode C, et al. Randomized trial of daclatasvir and asunaprevir with or without PegIFN/RBV for hepatitis C virus genotype 1 null responders. J Hepatol. 2014;60:490-9. 
7. Suzuki Y, Ikeda K, Suzuki F, et al. Dual oral therapy with daclatasvir and asunaprevir for patients with HCV genotype $1 \mathrm{~b}$ infection and limited treatment options. J Hepatol. 2013;58:655-62.

8. Kumada H, Suzuki Y, Ikeda K, et al. Daclatasvir plus asunaprevir for chronic HCV genotype $1 \mathrm{~b}$ infection. Hepatology. 2014;59:2083-91.

9. Manns M, Pol S, Jacobson IM, et al. All-oral daclatasvir plus asunaprevir for hepatitis $\mathrm{C}$ virus genotype 1b: a multinational, phase 3 , multicohort study. Lancet. 2014;384:1597-605.

10. Chayama K, Suzuki F, Suzuki Y, et al. All-oral dual combination of daclatasvir plus asunaprevir compared with telaprevir plus peginterferon alfa/ ribavirin in treatment-naive Japanese patients chronically infected with HCV genotype 1b: results from a phase 3 study. Hepatology. 2014;60(suppl):1135A.

11. Alter MJ. Epidemiology of hepatitis $C$ virus infection. World J Gastroenterol. 2007;13:2436-41.

12. Wright TL. Treatment of patients with hepatitis C and cirrhosis. Hepatology. 2002;36:S185-94.

13. Iwasaki $\mathrm{Y}$, Ikeda $\mathrm{H}$, Araki $\mathrm{Y}$, et al. Limitation of combination therapy of interferon and ribavirin for older patients with chronic hepatitis C. Hepatology. 2006;43:54-63.

14. Pol S, Ghalib RH, Rustgi VK, et al. Daclatasvir for previously untreated chronic hepatitis $C$ genotype 1 infection: a randomised, parallel-group, doubleblind, placebo-controlled, dose-finding, phase $2 \mathrm{a}$ trial. Lancet Infect Dis. 2012;12:671-7.

15. McPhee F, Hernandez D, Yu F, et al. Resistance analysis of hepatitis $\mathrm{C}$ virus genotype 1 prior treatment null responders receiving daclatasvir and asunaprevir. Hepatology. 2013;58:902-11.

16. McPhee F, Hernandez D, Zhou N, et al. Virological escape in $\mathrm{HCV}$ genotype 1-infected patients receiving daclatasvir plus ribavirin and peginterferon alfa-2a or alfa-2b. Antivir Ther. 2014;19:479-90.

17. Karino Y, Toyota J, Ikeda K, et al. Characterization of virologic escape in hepatitis $C$ virus genotype $1 \mathrm{~b}$ patients treated with the direct-acting antivirals daclatasvir and asunaprevir. J Hepatol. 2013;58:646-54.

18. Ikeda K, Saitoh S, Kobayashi M, et al. Distinction between chronic hepatitis and liver cirrhosis in patients with hepatitis $\mathrm{C}$ virus infection. Practical discriminant function using common laboratory data. Hepatol Res. 2000;18:252-66.

19. Poordad F, Sievert W, Mollison L, et al. Fixed-dose combination therapy with daclatasvir, asunaprevir, and beclabuvir for noncirrhotic patients with HCV genotype 1 infection. JAMA. 2015;313:1728-35.

20. Muir AJ, Poordad F, Lalezari J, et al. Daclatasvir in combination with asunaprevir and beclabuvir for hepatitis C virus genotype 1 infection with compensated cirrhosis. JAMA. 2015;313:1736-44.

21. Sulkowski MS, Gardiner DF, Rodriguez-Torres M, et al. Daclatasvir plus sofosbuvir for previously treated or untreated chronic HCV infection. N Engl J Med. 2014;370:211-21.

22. Friborg J, Zhou N, Han Z, et al. In vitro assessment of re-treatment options for patients with Hepatitis C Virus genotype $1 \mathrm{~b}$ infection resistant to daclatasvir plus asunaprevir. Infect Dis Ther. 2015;4:137-44. 